\title{
УСТРАНЕНИЕ ТУПИКОВОЙ ВИБРАЦИИ ТРУБОПРОВОДНОЙ ОБВЯЗКИ КОМПРЕССОРНОГО ЦЕХА В УСЛОВИЯХ ЭКСПЛУАТАЦИИ
}

\author{
Бураншин Айдар Раисович',
} aidarburanshin@mail.ru

\section{Годовский Дмитрий Александрович', diingo1@mail.ru}

\author{
Токарев Артём Павлович', \\ art-tokarev@yandex.ru \\ 1 Уфимский государственный нефтяной технический университет, \\ Россия, 450062, г. Уфа, ул. Космонавтов, 1.
}

Актуальность исследования. В настоящее время растет роль мероприятий, направленных на повышение надежности оборудования при магистральном трубопроводном транспорте природного газа. К факторам, негативно влияющим на надежность эксплуатации оборудования компрессорных станций магистральных газопроводов, относится возникающая по различным причинам повышенная вибрация. Одной из таких причин является сочетание определенных геометрических размеров трубопроводных коммуникаций и высоких расходов технологического газа.

Цель: уточнить режимы работы компрессорного цеха с 8 полнонапорными агрегатами, позволяющие снизить интенсивность тупиковой вибрации, наблюдающейся в трубопроводной обвязке отключенных газоперекачивающих агрегатов.

Объект: участок трубопроводных коммуникаций компрессорных станций от коллектора циклонных пылеуловителей до входного коллектора компрессорного цеха, тройники с отводами к газоперекачиващим агрегатам, отключенными кранами № 1 от входного коллектора.

Методы: вибрационное диагностирование трубопроводной обвязки на существующей компрессорной станции; математическое моделирование режимов работы компрессорного цеха с применением численного решения (гидравлический расчет в сочетании с расчетом собственных частот и частот вынуждающей силы).

Результаты. Предложен способ устранения тупиковых колебаний в рамках эксплуатации, уточняющий требования нормативной документации. Разработана методика оценки условий возникновения тупиковых колебаний, которая основана на гидравлическом расчете кольцевого газопровода высокого давления с отборами. Рассмотрены два режима компрессорного цеха с 8 полнонапорными нагнетателями с производительностью в 100 млн м³/сут. на отстройку от резонанса. В результате проведенных расчетов с края компрессорного цеха уменьшено количество газоперекачивающих агрегатов, которые необходимо эксплуатировать для устранения тупиковых колебаний, по сравнению с рекомендациями нормативной документации. Это позволяет выводить в резерв второй газоперекачивающий агрегат с края компрессорного цеха и, следовательно, снизить скорость наработки данного газоперекачивающего агрегата при устранении повышенной тупиковой вибрации трубопроводной обвязки газоперекачивающего агрегата.

\section{Ключевые слова:}

Трубопроводная обвязка, гидравлический расчет, технологический трубопровод, тупиковые колебания, вибрация, пульсация газа, компрессорный цех.

\section{Введение}

Одним из факторов, приводящих к остановкам технологического процесса транспорта газа на компрессорных станциях (КС), является повышенная вибрация газоперекачивающих агрегатов (ГПА) и их трубопроводной обвязки (ТПО) (рис. 1).

Обзор литературы [2-9] показывает, что повышенная вибрация возникает благодаря источникам, имеющим как механическую, так и гидродинамическую природу. Согласно $[10,11]$, в технологических трубопроводах обвязки находящихся в резерве или в ремонте ГПА КС магистральных газопроводов (МГ) при определенном сочетании условий могут наблюдаться тупиковые колебания, источник которых имеет гидродинамическую природу.

Puс. 1. Факторы, приводящие к остановкам транспорта газа на компрессорных стациях магистральных газопроводов [1]

Fig. 1. Factors leading to stops of gas transport at compressor stations of main gas pipelines

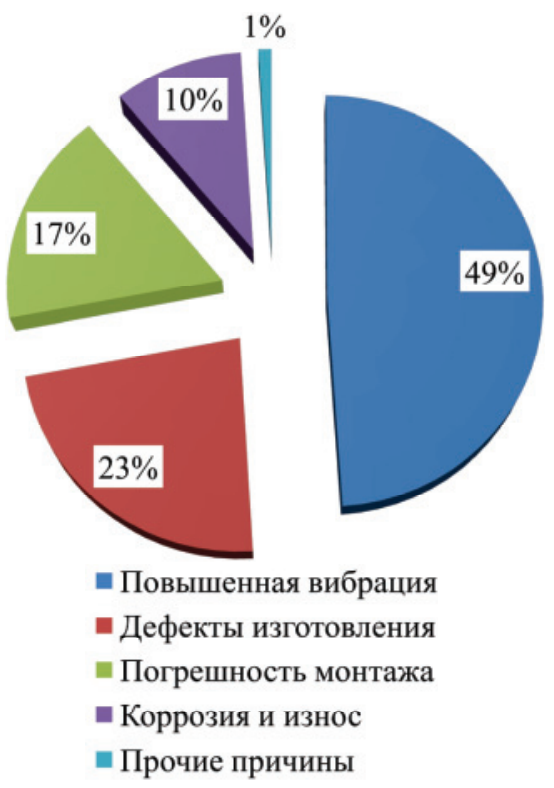

DOI 10.18799/24131830/2019/9/2268 


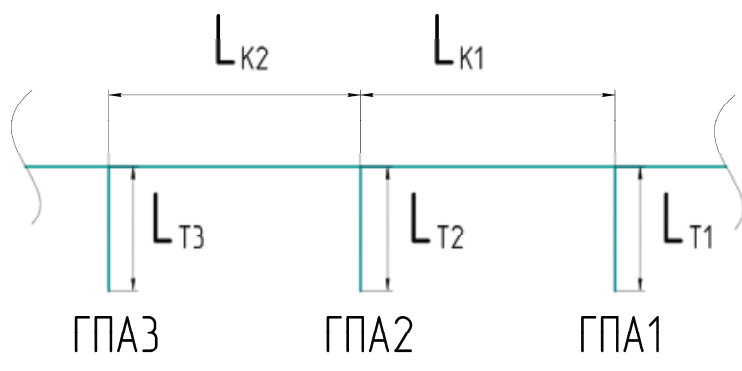

Pис. 2. Геолетрия трубопроводной обвязки компрессорного иеха

Fig. 2. Geometry of the piping of the compressor manufactory

Тупиковые колебания, согласно [12], - это акустические резонансные колебания. Данный вид вибрации называют тупиковой из-за характерного места проявления. Резонанс наблюдается при совпадении собственной акустической частоты газа в тупике с частотой, генерируемой в тройнике тупика расходного трубопровода-коллектора. Для их возникновения необходимо сочетание нескольких условий одновременно по геометрии трубопроводной обвязки и по скорости движения. Согласно [11], условиями по геометрии являются:

1) четное отношение длины коллектора между тройниками к средней длине тупиков (рис. 2);

$$
\frac{L_{\text {К.cp }}}{L_{\text {T.cp }}}=2,4,6 \ldots
$$

2) нечетное отношение длин тупиков.

$$
\frac{L_{\mathrm{T} .1}}{L_{\mathrm{T} .2}}=\frac{L_{\mathrm{T} .2}}{L_{\mathrm{T} .3}}=1,3,5 \ldots
$$

Условием по скорости [11] является превышение скорости потока газа некоторого критического значения:

$$
V_{\text {кр }}=\frac{D F_{\text {в }}}{S h},
$$

где $D$ - внутренний диаметр патрубка тройника со стороны подключения тупика; Sh - число Струхаля, для тройника на проход $0,25<\mathrm{Sh}<0,5[13,14]$; $F_{\text {в }}$ - частота пульсаций давления в тупике при прохождении потоком газа тройника (рис. 3), в случае резонанса она равняется собственной частоте акустических колебаний тупика $F_{\mathrm{c}}[11]$

$$
F_{\mathrm{B}}=F_{\mathrm{c}}=\frac{0,25 C_{3 \mathrm{~B}}(2 n-1)}{L_{\mathrm{T}}},
$$

где $C_{\text {вв }}$ - скорость звука в газе; $n=1,2,3 \ldots ; L_{\mathrm{T}}-$ длина тупика.

Повышенная вибрация, происходящая при тупиковых колебаниях в ТПО остановленных ГПА, оказывает отрицательное влияние на прочностные характеристики металла и в итоге на надежность и безопасность ответственного участка трубопровода технологического газа.

Для рассматриваемого вида вибрации требуется снизить уровень вибрации применением различных мероприятий, направленных на устранение причин возникновения тупиковых колебаний.

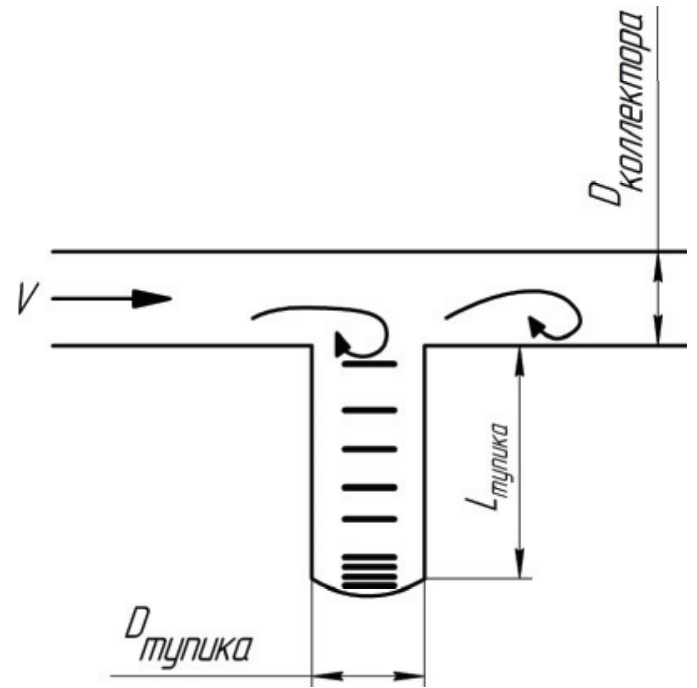

Pис. 3. Формирование пульсаций давления в тройнике на проход

Fig. 3. Formation of pressure pulsations in a tee on a passage

\section{Методы предотвращения повышенной тупиковой вибрации}

В литературе и нормативной документации $[12,15]$ предлагается два пути решения для устранения тупиковых колебаний:

1) реконструкция обвязки компрессорного цеха, что исключит условия возникновения вибрации по геометрии, т. е. изменится длина тупика и собственная частота акустических колебаний газа в нем. Как правило, мероприятие заключается в переносе кранов № 1. Также в ходе эксплуатации опытным путем было испытано, что открытие крана № 1 на остановленном ГПА при включенном маслонасосе уплотнения увеличивает длину тупика до обратного клапана перед краном № 2 и устраняет вибрацию в ТПО к данному ГПА.

2) ограничение режима работы компрессорного цеха, т. е. снижение общецеховой производительности или нахождение в работе двух-трех агрегатов (для компрессорного цеха с пятью-шестью агрегатами), находящихся с одной стороны компрессорного цеха (КЦ), как правило, наиболее близкой $\mathrm{k}$ коллектору пылеуловителей. При этом скорость газа далее во входном коллекторе снижается и не превышает критическую.

Последнее решение не позволяет выводить указанные агрегаты в ремонт или резерв, при этом наработка именно на этих агрегатах будет происходить быстрее. Что опять-таки приведет к скорому ремонту по наработке сразу двух или трех агрегатов.

Анализ тупиковых колебаний позволяет выделить две частоты: собственную частоту акустических колебаний тупика и частоту пульсаций давлений в тупике при прохождении тройника потоком газа.

В связи с резонансной природой вибрации, согласно требованиям для технологических трубо- 
проводов [16], необходима отстройка от резонанса, то есть совпадения частоты пульсаций давления $F_{\text {в }}$ генерируемых потоком газа при прохождении местного сопротивления - тройник на проход при определенной скорости с собственной частотой $F_{\text {c }}$ акустических колебаний газа в тупике [13].

Таким образом, можно опираться на нижнюю и верхнюю границы диапазона частот, при попадании в который будет наблюдаться повышенная вибрация [16]:

$$
0,75 \leq \frac{F_{\mathrm{B}}}{F_{\mathrm{c}}} \leq 1,3 .
$$

Далее будет использоваться нижняя граница этого диапазона в связи с невысокой скоростью движения газа и общим снижением скорости по ходу движения во входном коллекторе из-за отборов газа ГПА.

В работе изучена проблема повышенной низкочастотной вибрации в ТПО КЦ с 8 полнонапорными центробежными нагнетателями типа 235-21-1 с типовой обвязкой в соответствии с [17]. Анализ результатов виброобследования ТПО КЦ за 2016 г., свидетельствует о наличии повышенной тупиковой вибрации при определенных режимах работы КЦ на участке от тройника до крана № 1 в ТПО ГПА № 1-4, находящихся в резерве (рис. 4). В ТПО указанных агрегатов выполняются условия по геометрии для возникновения тупиковых колебаний. В ТПО ГПА № 5-8 указанные выше условия по геометрии не выполняются - иная длина тупика. $\mathrm{C}$ целью устранения тупиковых колебаний рассматриваются тройники только одного плеча КЦ с агрегатами № 1-4. Для решения данной проблемы необходимо определить условия возникновения повышенной вибрации при помощи анализа режимов работы КЦ и установления скоростей в тройниках входного коллектора, ведущих к ГПА № 1-4.

\section{Оценка условий возникновения тупиковых}

\section{колебаний и предложения по их устранению}

Для оценки условий возникновения тупиковых колебаний необходимо определить скорость движения газа и частоту вынуждающей силы. С це- лью определения скорости был выполнен гидравлический расчет участка кольцевого газопровода высокого давления с использованием формул из $[18,19]$.

Расчет газопровода, точнее кольцевого участка, образованного параллельными шлейфами и коллекторами пылеуловителей и входного для компрессорного цеха (рис. 4), затруднен вследствие различных режимов и производительностей компрессорного цеха и значительного влияния местных сопротивлений.

При движении газа по трубопроводу происходит снижение давления вследствие преодоления сил трения по длине и на местных сопротивлениях, таких как поворот на 90 градусов, тройники на проход, на разделение и слияние.

Для облегчения расчета потери на местные сопротивления заменяются потерями по длине на эквивалентном участке:

$$
L_{\text {экв }}=\sum \xi \frac{D_{\mathrm{BH}}}{\lambda},
$$

где $\xi$ - коэффициент $i$-го местного сопротивления; $D_{\text {вн }}-$ внутренний диаметр; $\lambda-$ коэффициент гидравлического сопротивления

В работе для упрощения расчета предложена замена кольцевого газопровода на два параллельных с отборами, а их, в свою очередь, - на газопроводы с постоянным расходом [20]. Предполагается, что конечная точка выделенных параллельных газопроводов находится в тройнике к рабочему ГПА. При четырех работающих ГПА - в третьем по счету работающем ГПА, ведя отсчет по наиболее короткому шлейфу, при пяти ГПА - в центральном работающем ГПА. Также примем, что расход центробежных нагнетателей одинаков.

В связи с малой длиной участка по сравнению с магистральными газопроводами изменением температуры по длине можно пренебречь и расчет проводить для газопровода с постоянной по длине температурой.

Проведем расчет для КЦ с 8 полнонапорными нагнетателями при работе ГПА № 4-8 (рис. 5). Длины участков приведены в табл. 1 .

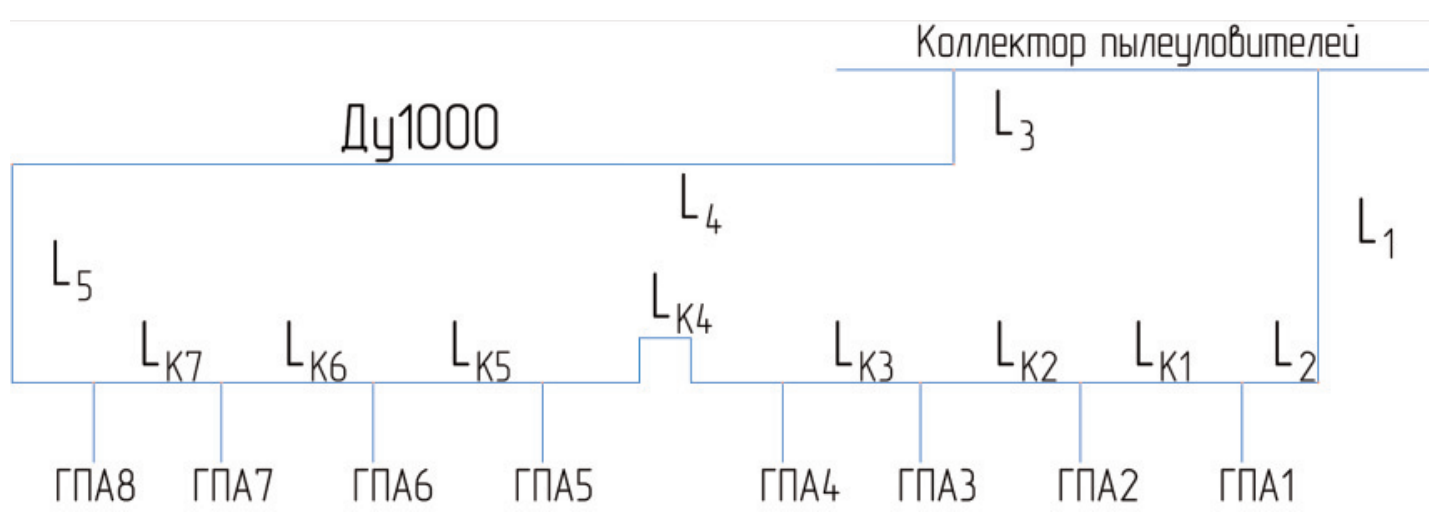

Pис. 4. Расчетная схема

Fig. 4. Calculation scheme 


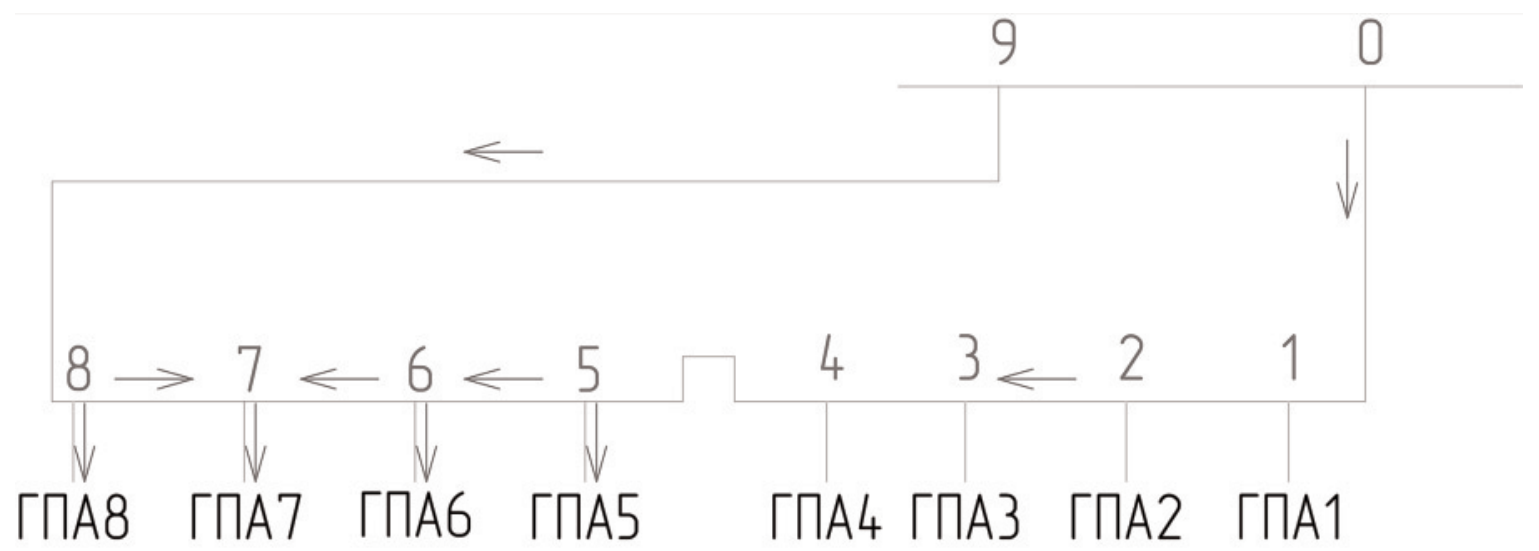

Рис. 5. Распределение потоков с обозначением участков

Fig. 5. Breakdown of flux with designation of pipe section

Таблица 1. Длины участков кольцевого газопровода

Table 1. Lengths of sections of the loop gas pipeline

\begin{tabular}{|l|c|c|c|}
\hline $\begin{array}{l}\text { Обозначение на схеме } \\
\text { Designation on the scheme }\end{array}$ & $L_{9-8}$ & $L_{8-7}$ & $L_{7-6}$ \\
\hline Длина, м/Length, m & 167,42 & 18 & 18 \\
\hline $\begin{array}{l}\text { Обозначение на схеме } \\
\text { Designation on the scheme }\end{array}$ & $L_{6-5}$ & $L_{5-4}$ & $L_{4-3}$ \\
\hline Длина, м/Length, m & 18 & 18,125 & 18,015 \\
\hline $\begin{array}{l}\text { Обозначение на cхеме } \\
\text { Designation on the scheme }\end{array}$ & $L_{3-2}$ & $L_{2-1}$ & $L_{1-0}$ \\
\hline Длина, м/Length, m & 19,77 & 18,03 & 44,02 \\
\hline
\end{tabular}

Расчет идет в следующем порядке:

1) Задаваясь некоторым отношением расходов по длинному и короткому шлейфу при известной цеховой производительности (в качества примера $100 \mathrm{млн}^{3} /$ сут), определим расход на каждом участке газопроводов с отборами (табл. 2).

2) По известным значениям расхода и геометрическим параметрам трубопроводов определяется режим течения и, соответственно, коэффициенты сопротивления трения и гидравлического сопротивления.

Таблица 2. Распределение расходов по участкам

Table 2. Breakdown of flow rate by pipe section

\begin{tabular}{|c|c|c|c|}
\hline \multicolumn{3}{|c|}{ Параллельный газопровод/Parallel gas pipeline } \\
\hline \multicolumn{2}{|c|}{ № 1 (0-6) } & \multicolumn{2}{c|}{ № 2 (9-6) } \\
\hline $\begin{array}{c}\text { Участок } \\
\text { Pipe section }\end{array}$ & $\begin{array}{c}\text { Расход, млн } \mathrm{M}^{3} / \text { сут } \\
\text { Flow rate, } \\
\text { million } \mathrm{m}^{3} \text { per day }\end{array}$ & $\begin{array}{c}\text { Участок } \\
\text { Pipe section }\end{array}$ & $\begin{array}{c}\text { Paсход, млн м³ } / \text { сут } \\
\text { Flow rate, } \\
\text { million } \mathrm{m}^{3} \text { per day }\end{array}$ \\
\hline $0-4$ & 50,40 & $9-8$ & 49,60 \\
\hline $4-5$ & 30,40 & $8-7$ & 29,60 \\
\hline $5-6$ & 10,40 & $7-6$ & 9,60 \\
\hline
\end{tabular}

Для квадратичного режима коэффициент сопротивления трения

$$
\lambda_{\text {тр }}=0,067\left(\frac{2 k_{\ni}}{D_{\text {вн }}}\right)^{0,2},
$$

где $k_{\text {э }}$ эквивалентная шероховатость.
Для смешанного режима

$$
\lambda_{\text {тр }}=0,067\left(\frac{158}{\operatorname{Re}}+\frac{2 k_{\ni}}{D_{\text {вн }}}\right)^{0,2},
$$

где $\operatorname{Re}$ - число Рейнольдса;

$$
\lambda=\frac{\lambda_{\text {тр }}}{\AA^{2}},
$$

где $E$ - коэффициент гидравлической эффективности, $E=0,95$.

3) Заменяются газопроводы с отборами на эквивалентные газопроводы с постоянным по длине значением расхода, согласно формулам [20].

$$
Q_{\text {әКв }}=\frac{\sum Q_{i} L_{i} \lambda_{i}}{L_{\text {эКв }} \lambda_{\text {экв }}} .
$$

4) По эквивалентным значениям расхода определяется при известном начальном давлении значение конечного давления для обоих параллельных газопроводов.

$$
P_{\text {к }}=\sqrt{P_{\mathrm{H}}^{2}-\frac{Q_{\text {экв }}^{2} \lambda_{\text {экв }} Z_{\mathrm{cp}} \Delta T_{\mathrm{cp}} L_{\text {экв }}}{K^{2} D_{\text {вн }}^{5}}},
$$

где $K$ - коэффициент, зависящий от размерности величин, при использовании смешанной системы единиц (МПа, км, К, млн м ${ }^{3} /$ сут, м) $K=105,087$.

5) Конечные давления в точке слияния потоков должны совпадать - при расхождении требуется уточнить распределение потоков по шлейфам и, в отдельных случаях, выбор конечной точки.

В связи с большим объемом расчетов и необходимостью уточнения распределения потоков при отклонениях значений конечных давлений расчет выполнен с использованием Microsoft Office Excel.

Согласно уточненному распределению потоков определяются расходы на каждом участке входного коллектора компрессорного цеха. Для приведения расхода к условиям входного коллектора требуется перейти от стандартных условий, для которых был определен коммерческий расход. По приведенному расходу при условиях коллектора ста- 
новится известно значение скорости в тройниках с безрасходным отводом, что позволяет определить частоту пульсаций потока газа в тупиковом ответвлении.

Сравнение полученной частоты пульсаций давления с частотой, соответствующей нижней границе отстройки от резонанса, позволяет судить о наличии или отсутствии повышенной низкочастотной вибрации (табл. 3).

Таблица 3. Результаты расчета при работе газоперекачивающих агрегатов № 4-8

Table 3. Results of calculation at operation of gas-pumping units № $4-8$

\begin{tabular}{|c|c|c|c|c|c|c|}
\hline 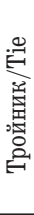 & 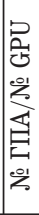 & 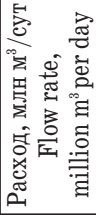 & 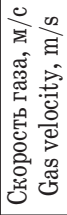 & $\begin{array}{c}\text { Частота } \\
\text { пульсаций } \\
\text { давления, Гц } \\
\text { Frequency of } \\
\text { pressure pul- } \\
\text { sations, Hz }\end{array}$ & $\begin{array}{c}\text { Нижняя грани- } \\
\text { ца отстройки } \\
\text { от резонанса } \\
\text { Lower limit } \\
\text { of detuning } \\
\text { from resonance }\end{array}$ & $\begin{array}{l}\text { Выполнение } \\
\text { отстройки } \\
\text { от резонанса } \\
\text { Realizing } \\
\text { tuning from } \\
\text { resonance }\end{array}$ \\
\hline 1 & 1 & 50,40 & 12,4 & 8,94 & 8,86 & $\begin{array}{l}\text { Не выполнена } \\
\text { Not performed }\end{array}$ \\
\hline 2 & 2 & 50,40 & 12,4 & 8,94 & 9,49 & $\begin{array}{c}\text { Выполнена } \\
\text { Performed }\end{array}$ \\
\hline 3 & 3 & 50,40 & 12,4 & 8,94 & 8,55 & $\begin{array}{l}\text { Не выполнена } \\
\text { Not performed }\end{array}$ \\
\hline 4 & 4 & 50,40 & 12,4 & 8,94 & 8,64 & $\begin{array}{c}\text { Выполнена } \\
\text { (ГПА в работе) } \\
\text { Performed (GPU } \\
\text { in operation) }\end{array}$ \\
\hline
\end{tabular}

Таблица 4. Результаты расчета при работе газоперекачивающих агрегатов № 1, 5-8

Table 4. Results of calculation at operation of gas-pumping units № 1, 5-8

\begin{tabular}{|c|c|c|c|c|c|}
\hline 承 & 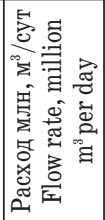 & 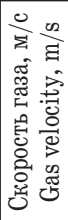 & $\begin{array}{c}\text { Частота } \\
\text { пульсаций } \\
\text { давления, Гц } \\
\text { Frequency of } \\
\text { pressure pul- } \\
\text { sations, Hz }\end{array}$ & \begin{tabular}{|} 
Нижняя граница \\
отстройки \\
от резонанса \\
Lower limit of \\
detuning from \\
resonance
\end{tabular} & $\begin{array}{c}\text { Выполнение } \\
\text { отстройки } \\
\text { от резонанса } \\
\text { Realizing tuning } \\
\text { from resonance }\end{array}$ \\
\hline 1 & 51,99 & 12,8 & 9,22 & 8,66 & $\begin{array}{c}\text { Выполнена } \\
\text { (ГПА в работе) } \\
\text { Performed } \\
\text { (GPU in operation) }\end{array}$ \\
\hline 2 & 31,99 & 7,9 & 5,68 & 9,49 & $\begin{array}{c}\text { Выполнена } \\
\text { Performed }\end{array}$ \\
\hline 3 & 31,99 & 7,9 & 5,68 & 8,55 & $\begin{array}{c}\text { Выполнена } \\
\text { Performed }\end{array}$ \\
\hline 4 & 31,99 & 7,9 & 5,68 & 8,64 & $\begin{array}{c}\text { Выполнена } \\
\text { Performed }\end{array}$ \\
\hline
\end{tabular}

По результатам расчетов (табл. 3) можно сделать вывод о наличии повышенной тупиковой вибрации в ТПО ГПА № 1, 3 при общецеховой произ-

\section{СПИСОК ЛИТЕРАТУРЫ}

1. Энтальцев С.В., Бердник А.Г. Разработка рекомендаций по проведению виброконтроля с целью повышения надежности технологического оборудования компрессорных станций // Трубопроводный транспорт 2018: Материалы XIII Междуна- водительности 100 млн м ${ }^{3} /$ сут и нахождении в резерве ГПА № 1-3.

Аналогичный расчет при рабочих ГПА № 1 , 5-8 показывает, что повышенная тупиковая вибрация не будет наблюдаться в ТПО остановленных ГПА № 2-4 (табл. 4).

\section{Заключение}

Анализ существующих методов снижения повышенной вибрации ТПО показал, что в рамках эксплуатации оперативно устранить условия для возникновения тупиковых колебаний можно регулированием работы КЦ. Согласно [12], ограничение работы заключается в снижении общецеховой производительности (уменьшается скорость потока газа во входном коллекторе в целом) или в нахождении в работе двух или трех ГПА, расположенных с края коллектора (уменьшается скорость в коллекторе после отводов к указанным ГПА), что приводит к ускоренной наработке данных ГПА.

Для оценки условий возникновения тупиковых колебаний (в связи с их газодинамической природой) в ТПО исследуемого КЦ рассмотрен кольцевой газопровод высокого давления с отборами, образованный выходным коллектором пылеуловителей и входным коллектором КЦ. Для его гидравлического расчета и определения скорости и частоты вынуждающей силы была разработана методика с использованием формул из $[16,17]$.

Построенная эмпирическая модель для сравнения действительной геометрии трубопроводной обвязки существующего КЦ и параметров потока транспортируемого газа в определенных тройниках с известными условиями возникновения тупиковых колебаний и выполненные расчеты сложного газопровода позволили определить параметры, соответствующие созданию пульсаций давления с частотой, близкой к собственной акустической частоте тупика.

Проведенное математическое моделирование для двух режимов работы КЦ позволяет сделать вывод об устранении тупиковой вибрации при определенных значениях производительности КЦ и конфигурации пяти работающих ГПА из восьми. Было уменьшено количество ГПА, расположенных с края КЦ, по результатам проведенных расчетов до одного по сравнению с общей рекомендацией [12] о двух работающих ГПА, расположенных с края КЦ. При этом уменьшается скорость наработки второго ГПА с края КЦ. Разработанная методика также дает возможность определить общецеховую производительность, при которой возможен вывод в ремонт/резерв первого ГПА с края КЦ.

родной учебно-научно-практической конференции. - Уфа. Изд-во УГНТУ, 2018. - С. 381-383.

2. Ashly H., Haviland G. Bending vibration of pipeline containing fluid // Journal of Applied Mechanics. - 1950. - V. 17. - № 3. P. 229-232. 
3. Baird R.C., Bechtold J.C. Mechanical vibration of piping induced by gas-pressure pulsation // Transactions of the ASME. - 1949. V. 71. - № 8. - P. 989-995.

4. Udoetok E.S. Internal Fluid Flow Induced Vibration of Pipes // Journal of Mechanical Design and Vibration. - 2018. - V. 6. № 1. - P. 1-8.

5. Czerwinski A., Luczko J. Vibration of Steel Pipes and Flexible Hoses Induced by Periodically Variable Fluid Flow // Mechanics and Control. - 2012. - V. 31. - № 2. - P. 63-71.

6. Faal R.T., Derakhshan D. Flow-Induced Vibration of Pipeline on Elastic Support // Procedia Engineering. - 2011. - V. 14. P. 2986-2993.

7. Paidoussis M. Review of flow-induced vibrations in reactors and reactors components // Journal of Nuclear Engineering and Design. - 1982. - V. 74. - № 1. - P. 31-60.

8. Zhang Y.L., Gorman D.G, Reese J.M. Analysis of the Vibration of Pipes Conveying Fluid // Proceeding Institution of Mechanical Engineers. - 1999. - V. 213. - P. 849-860.

9. Paidoussis M., Sundrajan C. Parametric and combination resonance of a pipe conveying pulsating fluid // Transaction of the ASME. - 1975. - V. 42. - № 4. - P. 780-784.

10. Ангалев А.М., Соколинский Л.И., Лопатин А.С. Исследования вибрации и пульсации газа в системах «центробежный нагнетатель - трубопровод» // Труды Российского государственного университета нефти и газа им. И.М. Губкина. - 2009. - № 4. C. $74-85$.

11. Обеспечение виброустойчивости технологических трубопроводов пускового контура КС / В.И. Акимов, Т.А. Бакиев, С.В. Скрынников, Л.И. Соколинский // Газовая промышленность. - 2015. - № 8. - С. 56-59.

12. СТО Газпром 2-2.3-324-2009. Диагностическое виброобследование технологических трубопроводов компрессорных цехов с центробежными нагнетателями. Нормы оценки и методы проведения работ. URL: https://www.twirpx.com/file/573981/ (дата обращения: 25.03.2018).

13. Якубович В.А. Основы вибродиагностики. - М.: ИТЦ ОРГТЕХДИАГНОСТИКА, 1998. - 37 с.

14. Якубович В.А., Горбунов С.Г. Вибрационный контроль и диагностика. - М.: МАКС Пресс, 2007. - 200 с.

15. Бураншин А.Р., Годовский Д.А., Токарев А.П. Устранение тупиковых колебаний обвязки центробежных компрессоров природного газа // 68-я научно-техническая конференция студентов, аспирантов и молодых ученых: Материал. конф. - Уфа: УГНТУ, 2017. - Кн. 1 - С. 394-395.

16. ГОСТ 32388-2013. Трубопроводы технологические. Нормы и методы расчета на прочность, вибрацию и сейсмические воздействия. - М.: Стандартинформ, 2014. - 114 с.

17. СТО Газпром 2-3.5-051-2006. Нормы технологического проектирования магистральных газопроводов. URL: http://files. stroyinf.ru/Data1/49/49848/ (дата обращения: 25.12.2018).

18. Ванчин А.Г. Простая гидравлическая модель компрессорного цеха магистрального газопровода // Нефтегазовое дело. 2013. - № 1. - C. 273-280.

19. Бураншин А.Р., Годовский Д.А., Токарев А.П. Определение режима работы компрессорного цеха в случае тупиковых колебаний // Трубопроводный транспорт 2018: Материалы XIII Международной учебно-научно-практической конференции. Уфа: Изд-во УГНТУ, 2018. - С. 383-384.

20. Коршак А.А., Нечваль А.М. Проектирование и эксплуатация газонефтепроводов / под ред. А.А. Коршак. - Ростов-на-Дону: Феникс, 2016. - 540 c.

Поступила 10.06.2019 2.

\section{Информация об авторах}

Бураншин A.P., магистр кафедры гидрогазодинамики трубопроводных систем и гидромашин, Уфимский государственный нефтяной технический университет.

Годовский Д.А., кандидат технических наук, доцент кафедры гидрогазодинамики трубопроводных систем и гидромашин, Уфимский государственный нефтяной технический университет.

Токарев А.П., доцент кафедры гидрогазодинамики трубопроводных систем и гидромашин, Уфимский государственный нефтяной технический университет. 


\title{
ELIMINATION OF DEAD-END OSCILLATIONS OF COMPRESSOR MANUFACTORY PIPING IN OPERATING CONDITIONS
}

\author{
Aydar R. Buranshin', \\ aidarburanshin@mail.ru \\ Dmitriy A. Godovskiy', \\ diingo1@mail.ru \\ Artem P. Tokarev', \\ art-tokarev@yandex.ru \\ 1 Ufa State Petroleum Technological University, \\ 1, Kosmonavtov street, Ufa, 450062, Russia.
}

The relevance of research. At present, the role of activities aimed to improving the reliability of equipment in the main pipeline transport of natural gas is growing. The factor that negatively affects the reliability of operation of compressor stations equipment of main gas pipelines is the increased vibration due to various reasons. One of these reasons is the combination of certain geometric dimensions of pipelines and high flow of process gas.

The aim of the research is to define the modes of a compressor manufactory with 8 full-pressure units to reduce the intensive deadend oscillation observed in off-cycle compressor units' piping.

Object: pipeline communications section of the compressor station from the cyclone dust collectors to the inlet manifold of the compressor manufactory, tees with branches to the gas-pumping unit, switched off by cranes No. 1 from the inlet manifold.

Methods: vibration diagnostics of piping at the existing compressor station; mathematical modeling of operating modes of the compressor manufactory using a numerical solution (hydraulic calculation in combination with the calculation of natural frequencies and frequencies of the driving force).

Findings. The paper proposes a method for eliminating dead-end oscillations within the scope of operation, specifying the requirements of regulatory documentation. The methodology for estimating the conditions of dead-end oscillations appearance is developed. It is based on the hydraulic calculation of a high-pressure loop gas pipeline with selections. Two modes of a compressor manufactory with 8 full-pressure units with a capacity of 100 million $\mathrm{m}^{3}$ /day are considered to detuning from resonance. As a result of the calculations, the number of gas-pumping units from the edge of the compressor manufactory, that must be operated to eliminate dead-end oscillations, has been reduced in comparison with the recommendations of normative documentation. This allows putting the second gas-pumping unit from the edge of the compressor manufactory into reserve and, consequently, reducing the operating time of this gas-pumping unit while eliminating the high dead-end oscillations of the piping.

\section{Key words:}

Piping, hydraulic calculation, process piping, dead-end oscillations, vibration, gas pulsation, compressor manufactory.

\section{REFERENCES}

1. Entaltsev S.V., Berdnik A.G. Razrabotka rekomendatsiy po provedeniyu vibrokontrolya s tselyu povysheniya nadezhnosti tekhnologicheskogo oborudovaniya kompressornykh stantsiy [Development of recommendations for vibration control to improve the reliability of compressor station equipment]. Truboprovodny transport 2018. Materialy XIII Mezhdunarodnoy uchebno-nauchnoprakticheskoy konferentsii [Pipeline transportation 2018. Materials of the XIII International Academic and Scientific-Practical Conference]. Ufa, UGNTU Publ., 2018. pp. 381-383.

2. Ashly H., Haviland G. Bending vibration of pipeline containing fluid. Journal of Applied Mechanics, 1950, vol. 17, no. 3, pp. 229-232.

3. Baird R.C., Bechtold J.C. Mechanical vibration of piping induced by gas-pressure pulsatione. Transactions of the ASME, 1949, vol. 71, no. 8, pp. 989-995.

4. Udoetok E.z. Internal Fluid Flow Induced Vibration of Pipes. Journal of Mechanical Design and Vibration, 2018, vol. 6, no. 1, pp. $1-8$.

5. Czerwinski A., Luczko J. Vibration of Steel Pipes and Flexible Hoses Induced by Periodically Variable Fluid Flow. Mechanics and Control, 2012, vol. 31, no. 2, pp. 63-71.

6. Faal R.T., Derakhshan D. Flow-Induced Vibration of Pipeline on Elastic Support. Procedia Engineering, 2011, vol. 14, pp. 2986-2993.
7. Paidoussis M. Review of flow-induced vibrations in reactors and reactors components. Journal of Nuclear Engineering and Design, 1982, vol. 74, no. 1, pp. 31-60.

8. Zhang Y.L., Gorman D.G, Reese J.M. Analysis of the Vibration of Pipes Conveying Fluid. Proceeding Institution of Mechanical Engineers, 1999, vol. 213, pp. 849-860.

9. Paidoussis M., Sundrajan C. Parametric and combination resonance of a pipe conveying pulsating fluid. Transaction of the $A S$ $M E, 1975$, vol. 42, no. 4, pp. 780-784.

10. Angalev A.M., Sokolinsky L.I., Lopatin A.S. Research of vibration and pulsation of gas in systems "centrifugal supercharger pipeline». Proceedings of the Gubkin Russian State University of Oil and Gas, 2009, no. 4, pp. 74-85. In Rus.

11. Akimov V.I., Bakiev T.A., Skrynnikov S.V., Sokolinsky L.I. Ensuring the vibration resistance of technological pipelines of the starting circuit of the compressor station. The gas industry, 2015 , no. 8, pp. 56-59. In Rus.

12. STO Gazprom 2-2.3-324-2009. Diagnosticheskoe vibroobsledovanie tekhnologicheskikh truboprovodov kompressornykh tsekhov s tsentrobezhnymi nagnetatelyami. Normy otsenki i metody provedeniya rabot [ST0 Gazprom 2-2.3-324-2009. Diagnostic vibratory examination of technological pipelines of compressor shops with centrifugal units. Norms of assessment and methods of work]. Available at: https://www.twirpx.com/file/573981/ (accessed: 25 March 2018). 
13. Yakubovich V.A. Osnovy vibrodiagnostiki [Fundamentals of vibrodiagnostics]. Moscow, ITC ORGTECHDIAGNOSTICA Publ., $1998.37 \mathrm{p}$.

14. Yakubovich V.A., Gorbunov S.G. Vibratsionnyy kontrol i diagnostika [Vibration control and diagnostics]. Moscow, MAX Press, 2007. $200 \mathrm{p}$.

15. Buranshin A.R., Godovskiy D.A., Tokarev A.P. Ustranenie tupikovykh kolebaniy obvyazki tsentrobezhnykh kompressorov prirodnogo gaza [Elimination of dead-end oscillations of the piping of centrifugal compressors of natural gas]. 68-ya nauchnotekhnicheskaya konferentsiya studentov, aspirantov $i$ molodykh uchenykh. Materialy konferentsii $\left[68^{\text {th }}\right.$ scientific and technical conference of students, graduate students and young scientists. Conf. Proc.]. Ufa, UGNTU Publ., 2017. B. 1, pp. 394-395.

16. GOST 32388-2013. Truboprovody tekhnologicheskie. Normy i metody rascheta na prochnost, vibratsiyu i seysmicheskie vozdeystviya [State Standart 32388-2013. Technological Pipelines. Norms and methods for calculating strength, vibration and seismic effects]. Moscow, StandardInform Publ., 2014. 114 p.

17. STO Gazprom 2-3.5-051-2006. Normy tekhnologicheskogo proektirovaniya magistralnykh gazoprovodov [STO Gazprom
2-3.5-051-2006. Norms of technological design of main gas pipelines.] Available at: http://files.stroyinf.ru/Data1/49/49848/ (accessed: 25 December 2018).

18. Vanchin A.G. A simple hydraulic model of the compressor shop of the main gas pipeline. Oil and gas, 2013, no. 1, pp. 273-280. In Rus.

19. Buranshin A.R., Godovskiy D.A., Tokarev A.P. Opredelenie rezhima raboty kompressornogo tsekha v sluchae tupikovykh kolebaniy [Determination of operating mode of the compressor shop in the case of dead-end oscillations]. Truboprovodny transport 2018. Materialy XIII Mezhdunarodnoy uchebno-nauchno-prakticheskoy konferentsii [Pipeline transportation 2018. Materials of the XIII International Academic and Scientific-Practical Conference]. Ufa, UGNTU Publ., 2018. pp. 383-384

20. Korshak A.A., Nechval A.M. Proektirovanie i ekspluatatsiya gazonefteprovodov [Design and operation of gas and oil pipelines]. Rostov-on-Don, Feniks Publ., 2016. 540 p.

Received: 10 June 2019.

\section{Information about the authors}

Aydar R. Buranshin, master, Ufa State Petroleum Technological University.

Dmitriy A. Godovskiy, Cand. Sc., associate professor, Ufa State Petroleum Technological University.

Artem P. Tokarev, associate professor, Ufa State Petroleum Technological University. 